\title{
Hamiltonian theory for vibrational line shapes of atoms adsorbed on surfaces
}

\author{
R. Guantes, J. L. Vega, and S. Miret-Artés \\ Instituto de Matemáticas y Física Fundamental, Consejo Superior de Investigaciones Científicas, \\ Serrano 123, 28006 Madrid, Spain \\ Eli Pollak \\ Chemical Physics Department, Weizmann Institute of Science, 76100 Rehovot, Israel
}

(Received 5 November 2003; accepted 10 March 2004)

\begin{abstract}
The vibrational motions of atomic adsorbates on surfaces can be probed by helium atom scattering. The experimental observable is the dynamic structure factor, which shows an inelastic peak around the vibrational frequency of the isolated adsorbates known as the frustrated translational or $T$-mode peak. In this paper we develop a theory for the line shape of this peak, as well as for its temperature-dependent shift and broadening, based on a Hamiltonian equivalent of the generalized Langevin equation. The theory can be used to infer physical parameters of the adatom-surface interaction, such as the friction coefficient, the barrier height to diffusion, and the anharmonicity parameter. Numerical simulations are used to ascertain the range of validity of the theory, which is also generalized to describe multidimensional systems and to include quantum corrections. We compare the theoretical predictions for the shift and broadening with experimental results for the $\mathrm{Na} / \mathrm{Cu}(001)$ system, showing quantitative agreement within experimental resolution. (C) 2004 American Institute of Physics. [DOI: 10.1063/1.1737299]
\end{abstract}

\section{INTRODUCTION}

Vibrational motions of atomic and molecular adsorbates on surfaces have been recently probed by the quasielastic $\mathrm{He}$ atom scattering (QHAS) technique. ${ }^{1-6}$ This experimental tool allows for an accurate determination of low-frequency vibrational modes, which are not accessible by other types of measurements such as infrared reflection adsorption spectroscopy (IRAS). ${ }^{5}$ A beam of low-energy He atoms is scattered by the adsorbates on the surface, changing momentum as a result of the collision process. If the ensemble of adsorbates is diffusing on the surface, a quasielastic peak is observed, centered at zero frequency and with a broadening proportional to the diffusion coefficient. If, in addition, some atoms or molecules are adsorbed on specific sites, an additional peak appears centered around the vibrational frequency of the adsorbate close to the bottom of the lateral potential well. This so-called $T$-mode peak provides information on the frictional damping experienced by the adsorbates due to electron-hole pair creation or phonon coupling to the metal substrate. ${ }^{7}$ It also provides details about the curvature and anharmonicity of the lateral potential-energy surface (PES) around the minima. ${ }^{1,8}$

For atomic adsorbates at low coverages, the analysis of the $T$-mode peak can be a direct measure of all these quantities, since only adsorbate-substrate interactions are present and there are no other internal or external vibrational modes coupled to the $T$-mode. The QHAS experiments present some well defined features of the $T$-mode: First, it is a dispersionless mode (independent on the parallel wave-vector transfer K) and of inelastic nature. Second, a Lorentzian profile provides a good fit for the $T$-mode line shape whenever it does not overlap with the quasielastic peak. Third, the loca- tion of the $T$-mode peak has been observed to shift linearly with temperature for several molecular ${ }^{2,4}$ and atomic ${ }^{3}$ systems. Fourth, the peak width has been observed to vary linearly with temperature for some adsorbate-surface systems, like $\mathrm{Na} / \mathrm{Cu}(001)$ (Ref. 3) or $\mathrm{CO} / \mathrm{Cu}(001),{ }^{2}$ while for other systems such as $\mathrm{CO}$ on $\operatorname{Pt}(111)$ (Ref. 4) it appears to be independent of the temperature.

Despite the fact that knowledge of the adsorbatesubstrate interaction potential and the vibrational damping is fundamental for understanding surface processes such as adsorbate diffusion $^{3,9}$ or the operation of lubricants on the atomic scale, ${ }^{10}$ very few theoretical studies have been aimed to extract information about these physical quantities directly from the line shape of the vibrational peaks, ${ }^{2,7,11}$ or to relate these features to the adsorbate dynamics. A simple explanation for the linear dependence of the broadening at high temperatures, assuming transitions from a Boltzmann population of vibrational levels of an anharmonic oscillator, has been given in Ref. 2. At the same time, extensive numerical simulations using a semiempirical PES for the adiabatic adatomsurface interaction demonstrated ${ }^{3}$ that a Langevin equation approach is able to accurately reproduce the experimental results, not only for the quasielastic peak but also for the shift and broadening of the $T$-mode peak. Persson ${ }^{7,11}$ also used a continuum Brownian motion model to analyze the temperature dependence of the infrared line shape of vibrational modes of $\mathrm{CO}$ chemisorbed on $\mathrm{Ni}(111)$. These results suggest that the line shape of low-frequency $T$-mode peaks can be analyzed within a stochastic classical framework, and the origin of the shift and broadening is the thermal noise combined with the anharmonicity of the potential around the well bottom. 


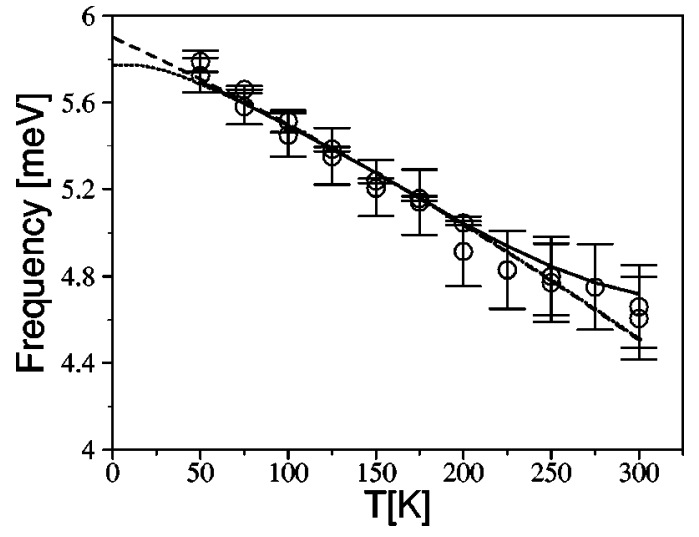

FIG. 1. T-mode peak position as a function of temperature for the $\mathrm{Na} /$ $\mathrm{Cu}(001)$ system. Circles: experimental results, Ref. 3. Solid line: numerical results obtained by solving the Langevin equation with the $1 \mathrm{D}$ cosine potential (3.1). The parameters used, $\gamma=0.1 \omega_{0}$ and $V^{\ddagger}=75 \mathrm{meV}$, have been estimated previously from experiment (Ref. 3). Dashed line: the theoretical estimate using second-order anharmonic corrections, Eq. (1.2). The dotted line is the theory using the quantum mechanical averages instead of the classical ones.

A central result of this paper is the derivation of simple analytic expressions for the temperature dependence of the peak location and width as functions of temperature. Specifically, to leading order, the $T$-mode potential of mean force has the generic form

$$
V(q)=\frac{1}{2} \omega_{0}^{2} q^{2}+K_{4} q^{4},
$$

where $q$ is the mass weighted $T$-mode vibrational coordinate, $\omega_{0}$ the harmonic frequency, and $K_{4}$ is the anharmonicity constant. To second order in the anharmonicity and provided that the damping constant $\gamma$ is not too large $\left(\gamma / \omega_{0} \leqslant 2\right)$ one finds for Ohmic friction, that the peak location $\left(\left\langle\lambda_{0}\right\rangle\right)$ is given by

$$
\left\langle\lambda_{0}\right\rangle=\sqrt{\omega_{0}^{2}-\frac{\gamma^{2}}{4}}+k_{B} T \frac{6 K_{4}}{\omega_{0}^{2} \sqrt{\omega_{0}^{2}-\frac{\gamma^{2}}{4}}}\left(1-k_{B} T \frac{9 K_{4}}{\omega_{0}^{4}}\right) .
$$

Here, $k_{B}$ is Boltzmann's constant and $T$ is the temperature. In Fig. 1, we plot this simple formula for the $\mathrm{Na} / \mathrm{Cu}(001)$ system and compare it with the experimental results. The parameters used have been estimated previously from experiment. ${ }^{3}$ Similarly, we find that for Ohmic friction the temperature and friction dependence of the width $(\sigma)$ of the $T$-mode peak is found to be

$$
\sigma=k_{B} T \frac{6\left|K_{4}\right|}{\omega_{0}^{2} \sqrt{\omega_{0}^{2}-\frac{\gamma^{2}}{4}}} .
$$

Using the same experimental parameters as for Fig. 1, we obtain the fit shown in Fig. 2 for the width. The detailed derivation of these expressions and their quantum mechanical extensions are given in Sec. IV below.

In addition to the derivation of these simple expressions, we will show that an analytic theory of the $T$-mode line

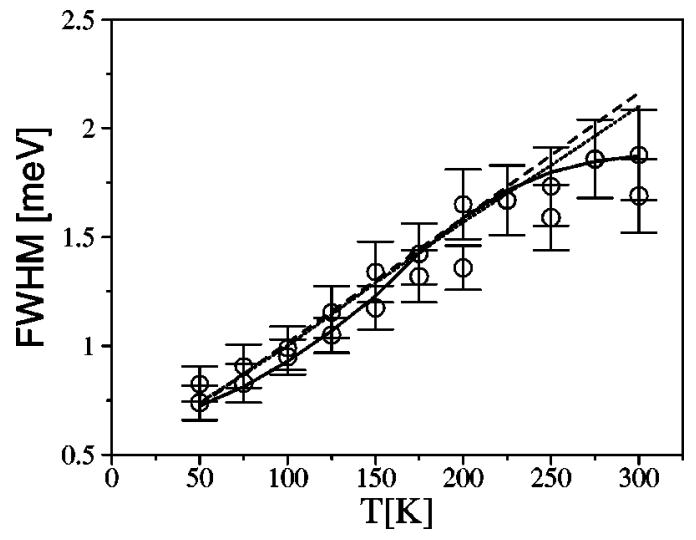

FIG. 2. $T$-mode full width at half maximum as a function of temperature for the $\mathrm{Na} / \mathrm{Cu}(001)$ system. Circles: experimental results, Ref. 3. Solid line: numerical results obtained as in Fig. 1. Dashed line: analytical estimate, Eq. (1.3). Dotted line: theoretical prediction, estimated by Fourier transforming the intermediate scattering function of the Kubo model, Eq. (4.5), see Sec. IV.

shapes may be derived from a microscopic Hamiltonian, thus including explicitly the adsorbate dynamics. In Sec. II we review the basic correlation functions needed for the analysis of QHAS experiments and the Hamiltonian formalism leading to a generalized Langevin equation. We will also provide the equations necessary for estimating the correlation functions within this formalism. In Sec. III we outline a perturbative theory of vibrational mode line shapes. We will examine the range of validity of the theory comparing it with numerical Langevin simulations. Extensions of the theory to the multidimensional case and quantum corrections are also investigated. Then in Sec. IV we derive Eqs. (1.2) and (1.3) from the Hamiltonian formalism. Finally, we end up with conclusions and a discussion relating our results to other experimental systems studied by QHAS techniques.

\section{BASIC THEORY}

\section{A. The quasielastic helium scattering formalism}

For simplicity, we will assume first that the adiabatic interaction potential is effectively one dimensional. The experimental observable is the dynamic structure factor $S(K, \omega)$, which is the Fourier transform in space and time of the distribution function $G(x, t) \cdot{ }^{12,13}$ At low adatom concentrations, interactions between adsorbates can be ignored, and $G(x, t)$ is defined as the probability of finding a single adatom at the position $x$ at time $t$, given that it was at the origin at some arbitrary time $t=0$. It is convenient to express the dynamic structure factor directly in terms of the intermediate scattering function $I(K, t)$, which is just the characteristic function of the position $x$ considered as a stochastic process:

$$
S(K, \omega)=\int_{-\infty}^{\infty} e^{-i \omega t} I(K, t) d t
$$

Here, $K$ is the wave-vector transfer of the He beam and the function $I(K, t)$ is defined by

$$
I(K, t)=\left\langle e^{-i K[x(t)-x(0)]}\right\rangle=\left\langle e^{-i K \int_{0}^{t} v\left(t^{\prime}\right) d t^{\prime}}\right\rangle .
$$


A cumulant expansion to second order yields the standard result ${ }^{14}$

$I(K, t) \sim e^{-K^{2} / 2 \int_{0}^{t} d t^{\prime} \int_{0}^{t} d t^{\prime \prime}\left\langle v\left(t^{\prime}\right) v\left(t^{\prime \prime}\right)\right\rangle}=e^{-K^{2} \int_{0}^{t}\left(t-t^{\prime}\right) C_{v}\left(t^{\prime}\right) d t^{\prime}}$,

where $C_{v}(t) \equiv\langle v(t) v(0)\rangle$ is the velocity autocorrelation function. The first approximation comes from the truncation of the cumulant series, and the second equality holds if the velocity process $v(t)$ is stationary. For Gaussian stochastic processes Eq. (2.3) is exact.

If the position process $x(t)$ is diffusive, then at times $t$ much longer than the correlation time,

$$
\int_{0}^{t}\left(t-t^{\prime}\right) C_{v}\left(t^{\prime}\right) d t^{\prime} \simeq t \int_{0}^{\infty} C_{v}\left(t^{\prime}\right) d t^{\prime}
$$

and the intermediate scattering function takes the simple form

$$
I(K, t)=e^{-K^{2} t / \tau_{c}}
$$

with

$$
\tau_{c}^{-1} \equiv \int_{0}^{\infty}\langle v(t) v(0)\rangle d t
$$

From Eq. (2.1) it is readily seen that the dynamic structure factor has a Lorentzian shape with a full width at half maximum (FWHM), $\Gamma=2 K^{2} \tau_{c}^{-1}$. Moreover, the righthand side of Eq. (2.6) is the Green-Kubo relation for the diffusion coefficient, thus $\Gamma=2 K^{2} D$. For diffusion at long times (or, analogously, small values of the wave-vector transfer $K$ ) the adiabatic potential plays almost no role and the position process can be considered as Gaussian. Therefore this FWHM reproduces quite accurately the behavior of the dynamic structure factor at small $K$, as has been observed experimentally and numerically. ${ }^{3,13,15}$ At higher values of the wave-vector transfer this is no longer a good approximation and other theoretical methods should be used to analyze the quasielastic peak and the diffusion process. In particular, Kramers' turnover theory has been recently shown to be a very convenient tool for studying activated surface diffusion. $^{9}$

On the other hand, the $T$-mode peak is the result of probing vibrational motions of the adsorbates. Typically, correlation times for the vibrational dynamics are much shorter than those for diffusive dynamics, and the two time scales can be separated. ${ }^{16}$ Also, the adiabatic atom-surface interaction can be expanded around the minimum of the well. For a symmetric surface, the first term gives a purely parabolic potential, and the position process $x(t)$ is the solution of a Langevin equation for a harmonic oscillator subject to Gaussian white noise and dissipation, which is a Gaussian stochastic process. ${ }^{17}$ Anharmonic corrections should account for the shift and broadening of the inelastic peak, whenever the Gaussian approximation remains valid.

\section{B. Hamiltonian formalism}

The position of an adatom of mass $\mu$ on a surface can be considered as a stochastic process obeying a generalized Langevin equation of the form (in mass weighted coordinates $q=\sqrt{\mu} x$ )

$$
\ddot{q}+\frac{\partial V(q)}{\partial q}+\int_{0}^{t} d \tau \gamma(t-\tau) \dot{q}(\tau)=F_{r}(t),
$$

where $V(q)$ is the adiabatic interaction potential and $F_{r}(t)$ the random force coming from a phonon bath. It is well known $^{18,19}$ that a Langevin equation of this form is equivalent to the Hamiltonian

$$
H=\frac{p_{q}^{2}}{2}+V(q)+\sum_{j=1}^{N}\left[\frac{p_{x_{j}}^{2}}{2}+\frac{1}{2}\left(\omega_{j} x_{j}-\frac{c_{j}}{\omega_{j}} q\right)^{2}\right],
$$

where the $j$ th harmonic bath mode is characterized by the mass weighted coordinate $x_{j}$, momentum $p_{x_{j}}$, and frequency $\omega_{j}$. The solution of Hamilton's equations of motion for the bath modes are expressed in terms of the system coordinate $q$ and the initial conditions, and then introduced into the equation of motion for the system variables $q, p_{q} \cdot{ }^{19}$ This leads to the Langevin equation (2.7) with the identification for the friction function

$$
\gamma(t)=\sum_{j=1}^{N} \frac{c_{j}^{2}}{\omega_{j}^{2}} \cos \left(\omega_{j} t\right) .
$$

For a suitably defined thermal distribution of initial conditions $^{19} F_{r}(t)$ is Gaussian distributed with zero mean and obeys the fluctuation dissipation theorem

$$
\left\langle F_{r}(t) F_{r}(0)\right\rangle=k_{B} T \gamma(t) .
$$

The continuum limit in the Hamiltonian formalism can be achieved using the spectral density given by ${ }^{18}$

$$
J(\omega)=\frac{\pi}{2} \sum_{j=1}^{N} \frac{c_{j}^{2}}{\omega_{j}}\left[\delta\left(\omega-\omega_{j}\right)-\delta\left(\omega+\omega_{j}\right)\right] .
$$

The Hamiltonian formulation presents some advantages over the phenomenological Langevin formulation: first of all, Ohmic and memory friction are treated in the same way, and the results obtained can be extended to any kind of friction function obeying the fluctuation dissipation relation (2.10). Second, the quantum treatment is straightforward, all that is needed is to treat the Hamiltonian [Eq. (2.8)] as an operator in the relevant Hilbert space. ${ }^{19}$

If the interaction potential $V(q)$ is purely harmonic, $V(q)=\omega_{0}^{2} q^{2} / 2$, the Hamiltonian (2.8) may be separated via a normal mode transformation. ${ }^{20,21}$ The normal-mode form is

$$
H_{\mathrm{NM}}=\frac{p_{\rho}^{2}}{2}+\frac{1}{2} \lambda_{0}^{2} \rho^{2}+\sum_{j=1}^{N}\left(\frac{p_{y_{j}}^{2}}{2}+\frac{1}{2} \lambda_{j}^{2} y_{j}^{2}\right),
$$

where the normal-mode coordinates of the system, $\rho$, and bath modes $y_{j}$ are related to the mass weighted coordinates $q$ and $x_{j}$ by an orthogonal transformation matrix $\mathbf{U}$. In particular, 


$$
q=u_{00} \rho+\sum_{j=1}^{N} u_{j 0} y_{j}
$$

The new system and bath frequencies, $\lambda_{0}$ and $\lambda_{j}$, may be expressed in terms of the old frequencies $\omega_{0}$ and $\omega_{j} \cdot{ }^{21} \mathrm{It}$ is convenient to define also a normal-mode friction function $^{22,23}$

$$
K(t)=\sum_{j=0}^{N} u_{j 0}^{2} \cos \left(\lambda_{j} t\right)
$$

and a normal-mode spectral density

$$
\Upsilon(\lambda)=\frac{\pi}{2} \sum_{j=0}^{N} \frac{u_{j 0}^{2}}{\lambda_{j}}\left[\delta\left(\lambda-\lambda_{j}\right)-\delta\left(\lambda+\lambda_{j}\right)\right],
$$

where the index 0 refers to the "system" normal mode $\rho$. The normal-mode transformation has the property that for any $s,{ }^{23}$

$$
\sum_{j=0}^{N} \frac{u_{j 0}^{2}}{s^{2}+\lambda_{j}^{2}}=\frac{1}{s^{2}+s \hat{\gamma}(s)+\omega_{0}^{2}},
$$

where $\hat{\gamma}(s)$ is the Laplace transform of the friction function. From this relationship, using the Fourier decomposition of the Dirac delta function, one can deduce the continuum limit for the normal-mode spectral density:

$$
\lambda \Upsilon(\lambda)=\operatorname{Re}[\hat{K}(i \lambda)]=\operatorname{Re}\left[\frac{i \lambda}{\omega_{0}^{2}-\lambda^{2}+i \lambda \hat{\gamma}(i \lambda)}\right] .
$$

These identities will be employed later on.

For future reference, we also note that the system normal-mode frequency $\lambda_{0}$ and the coefficient $u_{00}$ can be related to the friction function by the expressions ${ }^{21,24}$

$$
\begin{aligned}
& \lambda_{0}^{2}=\omega_{0}^{2}\left(1+\frac{\hat{\gamma}\left(i \lambda_{0}\right)}{i \lambda_{0}}\right)^{-1}, \\
& u_{00}^{2}=\left[1+\frac{1}{2}\left(\frac{\bar{\gamma}\left(i \lambda_{0}\right)}{i \lambda_{0}}+\left.\frac{d \hat{\gamma}(s)}{d s}\right|_{s=i \lambda_{0}}\right)\right]^{-1} .
\end{aligned}
$$

As is clear from Eq. (2.18), $\lambda_{0}$ is in general a complex frequency. The real part gives the frequency shift and the imaginary part the rate of energy transfer to the bath. For instance, for Ohmic friction $[J(\omega)=\gamma \omega]$ one sees that

$$
\lambda_{0}=\omega_{1}+i \gamma / 2
$$

with

$$
\omega_{1} \equiv \sqrt{\omega_{0}^{2}-\frac{\gamma^{2}}{4}} .
$$

As is well known, for a harmonic oscillator, with Ohmic dissipation, the oscillator frequency decreases with increasing damping constant but is independent of the temperature.

\section{VIBRATIONAL LINESHAPES}

\section{A. Harmonic oscillator}

A simple representation of the atom-surface interaction potential for a symmetric surface is the cosine form, ${ }^{29}$

$$
V(q)=V_{0}\left[1-\cos \left(\frac{2 \pi}{a} q\right)\right],
$$

where $a$ is the lattice spacing and $V_{0}$ determines the barrier height, $V^{\ddagger}=2 V_{0}$. The first term of a Taylor expansion around $q=0$ gives a parabolic potential with oscillator frequency

$$
\omega_{0}=2 \pi \sqrt{\frac{V_{0}}{\mu a^{2}}} .
$$

As stated above, for the harmonic oscillator case Eq. (2.3) is exact. The normal mode transformation is also exact. In normal-mode coordinates, the velocity autocorrelation function is

$$
\begin{aligned}
C_{v}(t)= & \left\langle\left(u_{00} p_{\rho}(t)+\sum_{j=1}^{N} u_{j 0} p_{y_{j}}(t)\right)\right. \\
& \left.\cdot\left(u_{00} p_{\rho}(0)+\sum_{j=1}^{N} u_{j 0} p_{y_{j}}(0)\right)\right\rangle .
\end{aligned}
$$

The equations of motion for the normal modes $y_{j}$ are those of a harmonic oscillator with frequency $\lambda_{j}$, so that

$$
y_{j}(t)=y_{j}(0) \cos \left(\lambda_{j} t\right)+\frac{p_{y_{j}}(0)}{\lambda_{j}} \sin \left(\lambda_{j} t\right) .
$$

The initial positions $y_{j}(0)$ and velocities $p_{y_{j}}(0)$ of the bath modes are distributed thermally, i.e.,

$$
\left\langle\lambda_{j}^{2} y_{j}^{2}(0)\right\rangle=\left\langle p_{j}^{2}(0)\right\rangle=\beta^{-1},
$$

where $\beta \equiv 1 / k_{B} T$.

Differentiating Eq. (3.4) with respect to time and using the thermal averages (3.5) one obtains

$$
C_{v}(t)=\frac{1}{\beta} K(t)
$$

Carrying out the inverse Laplace transform of Eq. (2.17) for Ohmic friction $[\hat{\gamma}(s)=\gamma]$, gives the standard result for the velocity autocorrelation function of the harmonic oscillator, ${ }^{17}$

$$
C_{v}(t)=\frac{e^{-(\gamma / 2) t}}{\beta}\left[\cos \left(\omega_{1} t\right)-\frac{\gamma}{2 \omega_{1}} \sin \left(\omega_{1} t\right)\right] .
$$

Substituting the exact harmonic velocity autocorrelation function, Eq. (3.7), in Eq. (2.3) for the intermediate scattering function gives

$$
\begin{aligned}
& I(K, t)=e^{-2 W} \cdot e^{2 W f_{0}(t)}, \\
& f_{0}(t)=e^{-\gamma t / 2}\left[\cos \left(\omega_{1} t\right)+\frac{\gamma}{2 \omega_{1}} \sin \left(\omega_{1} t\right)\right]
\end{aligned}
$$

and $2 W \equiv K^{2} / \mu \beta \omega_{0}^{2}$. We note that $2 W$ is the Debye-Waller attenuation factor, ${ }^{16}$ since

$$
2 W=K^{2}\left\langle q(t)^{2}+q(0)^{2}\right\rangle / 2 .
$$

The second exponential in Eq. (3.8) can be expanded as a power series in the variable $2 W$. The line shape is then obtained by Fourier transformation as in Eq. (2.1). Neglecting nonresonant terms one finds 


$$
\begin{aligned}
S(K, \omega) \simeq & e^{-2 W} \sum_{j} \frac{2^{j} W^{j}}{(j-1) !}\left(\frac{\gamma}{\left(\omega+\omega_{1} j\right)^{2}+\gamma^{2} j^{2} / 4}\right. \\
& \left.+\frac{\gamma}{\left(\omega-\omega_{1} j\right)^{2}+\gamma^{2} j^{2} / 4}\right) .
\end{aligned}
$$

This is a series of Lorentzians centered at the frequencies $\pm \omega_{1}$ and their harmonics, with decreasing intensities (typically only the case $j=1$ can be numerically or experimentally observed). The width of the first peak is the friction coefficient $\gamma$. Note also that the center is not at the oscillator frequency $\omega_{0}$ but at $\omega_{1}$; this is due to the coupling to the bath. Experimentally, the value of the friction coefficient is usually estimated by extrapolation of the width of the $T$ mode to zero temperature, a procedure which is justified by Eq. (3.10).

\section{B. Anharmonic corrections}

The full system (cosine) potential is approximated by its first anharmonic correction term, such that the system-bath Hamiltonian [Eq. (2.8)] may be expressed in terms of the normal modes as

$$
H=H_{\mathrm{NM}}+K_{4}\left(u_{00} \rho+\sum_{j=1}^{N} u_{j 0} y_{j}\right)^{4},
$$

where $H_{\mathrm{NM}}$ is the normal-mode Hamiltonian (2.12), and for the cosine potential $(3.1) K_{4} \equiv V^{(4)}(0) / 24 \mu^{2}=-\omega_{0}^{4} / 24 V_{0}$ is negative.

The time dependence for the normal-mode coordinates is solved by perturbation theory, to leading order in $K_{4}$ one has

$$
y_{j}(t)=y_{j}^{(0)}(t)+4 K_{4} y_{j}^{(1)}(t),
$$

where $y_{j}^{(0)}(t)$ is the harmonic oscillator solution given in Eq. (3.4), and the first-order correction $y_{j}^{(1)}(t)$ is

$$
y_{j}^{(1)}(t)=-\frac{u_{j 0}}{\lambda_{j}} \int_{0}^{t} \sin \left[\lambda_{j}\left(t-t^{\prime}\right)\right]\left(\sum_{j=0}^{N} u_{j 0} y_{j}^{(0)}\left(t^{\prime}\right)\right)^{3} d t^{\prime} .
$$

To calculate the velocity autocorrelation function we use the thermal averages

$$
\left\langle y_{j}^{2}(0) \frac{p_{k}^{2}(0)}{\lambda_{k}}\right\rangle=\left\langle\frac{p_{j}^{2}(0)}{\lambda_{j}^{2}} \frac{p_{k}^{2}(0)}{\lambda_{k}}\right\rangle=\frac{1}{\beta^{2} \lambda_{j}^{2} \lambda_{k}} .
$$

We remark that strictly one should use the total anharmonic Hamiltonian for the averaging, while Eq. (3.14) results from averaging with the normal-mode Hamiltonian. However, for the parameter range used later in this paper, this static correction is small and so can be neglected. The result for the velocity autocorrelation function is

$C_{v}(t)=\frac{1}{\beta} K(t)-\frac{12 K_{4}}{\beta^{2} \omega_{0}^{2}} \int_{0}^{t} K\left(t-t^{\prime}\right) \cdot \int_{0}^{t^{\prime}} K\left(t^{\prime \prime}\right) d t^{\prime \prime}$.

The first term is the harmonic oscillator contribution, Eq. (3.6), while for the second term is a convolution integral with the normal-mode friction function $K(t)$ [see Eq. (2.14)]. Laplace transformation, using the identity (2.16) yields the result

$$
\hat{C}_{v}(s)=\frac{1}{\beta} \frac{s}{s^{2}+s \hat{\gamma}(s)+\omega_{0}^{2}}-\frac{12 K_{4}}{\beta^{2} \omega_{0}^{2}} \frac{s}{\left[s^{2}+s \hat{\gamma}(s)+\omega_{0}^{2}\right]^{2}} .
$$

For Ohmic friction, inverse Laplace transformation gives the first anharmonic correction to the harmonic oscillator autocorrelation function as

$$
\begin{aligned}
C_{v}(t)= & C_{v}^{(0)}(t)-\frac{12 K_{4}}{\beta^{2} \omega_{0}^{2}} \\
& \times \frac{e^{-(\gamma / 2) t}\left[\gamma \omega_{1} t \cos \left(\omega_{1} t\right)+\left(2 \omega_{1}^{2} t-\gamma\right) \sin \left(\omega_{1} t\right)\right]}{4 \omega_{1}^{3}},
\end{aligned}
$$

where $C_{v}^{(0)}(t)$ is given in Eq. (3.7).

From Eq. (2.3), the intermediate scattering function can be then written as

$$
I(K, t)=I^{(0)}(K, t) \cdot I^{(1)}(K, t) .
$$

Here $I^{(0)}(K, t)$ is the harmonic oscillator scattering function, Eq. $(3.8), I^{(1)}(K, t)$ the first-order anharmonic correction for the cosine potential is

$$
I^{(1)}(K, t)=e^{-2 W / \beta V^{\ddagger}} \cdot e^{\left(2 W / \beta V^{\ddagger}\right) f_{1}(t)}
$$

and

$$
\begin{aligned}
f_{1}(t)= & e^{-\gamma t / 2}\left[\left(1-\frac{\gamma \omega_{0}^{2}}{4 \omega_{1}^{2}} t\right) \cos \left(\omega_{1} t\right)\right. \\
& \left.+\left(\frac{6 \gamma \omega_{0}^{2}-\gamma^{3}}{8 \omega_{1}^{3}}+\frac{\omega_{0}^{2}}{2 \omega_{1}} t\right) \sin \left(\omega_{1} t\right)\right] .
\end{aligned}
$$

Before comparing with numerical calculations we note that apart from the purely sinusoidal terms in the exponent similar to those appearing in Eq. (3.8), there are terms which are linear in $t$. Proceeding in the same way as for the derivation of Eq. (3.10), we see that they lead to Fano profiles centered at the harmonic frequencies. This causes a skewness of the $T$-mode peak changing its width and position. Second, the magnitude of the first-order correction depends on the reduced barrier height $\beta V^{\ddagger}$. For $\beta V^{\ddagger} \gg 1$ the harmonic approximation remains valid. This is expected since at low temperatures and high barriers (high vibrational frequencies) the particle remains close to the bottom of the potential well.

In order to establish the range of validity of the theory based on the leading first-order correction, we have solved numerically the Langevin equation (2.7) with Ohmic friction. The parameters are taken so as to model an adsorbed $\mathrm{Na}$ particle moving on a cosine potential, a system which has been investigated both theoretically ${ }^{27,28}$ and experimentally. ${ }^{29}$ In Fig. 3 we show the numerically determined velocity autocorrelation function for this system at two friction values: low, $\gamma=0.1 \omega_{0}$, and moderate, $\gamma=0.5 \omega_{0}$, and two different barrier heights $V^{\ddagger}$, compared to the harmonic and anharmonic approximations, Eqs. (3.7) and (3.17). As expected, doubling the barrier height (we could as well reduce the temperature) improves the agreement between the numerical and analytical autocorrelation functions. Note that the anharmonic correction shifts the frequency of oscillations 

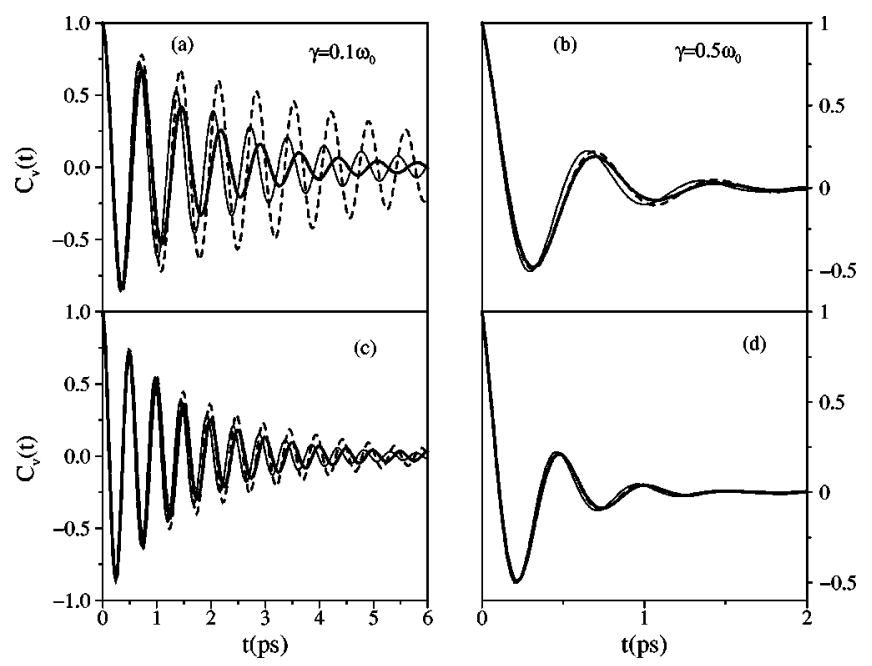

FIG. 3. Numerical and analytical velocity autocorrelation functions for a $\mathrm{Na}$ adsorbate on a cosine potential at $T=100 \mathrm{~K}$, with different friction coefficients $\gamma$ and barrier height values $V^{\ddagger}$. (a)-(c) $\gamma=0.1 \omega_{0} . V^{\ddagger}=67$ and 134 meV, respectively. (b)-(d) $\gamma=0.5 \omega_{0} . V^{\ddagger}=67$ and $134 \mathrm{meV}$. Thick solid line: numerical autocorrelation function. Thin solid line: harmonic approximation, Eq. (3.7). Dashed line: anharmonic correction, Eq. (3.17).

towards the correct values, but at low barrier and friction, Fig. 3(a), it fails to reproduce properly more than the first two or three peaks. Clearly, the first anharmonic correction does not suffice in this case. At higher friction, panels (b) and (d), the particle remains closer to the well bottom, and the anharmonic approximation reproduces properly the velocity autocorrelation function.

This can be also appreciated in the line shape (dynamic structure factor) shown in Fig. 4 at different temperatures. For comparison, we also plot with dashed lines the harmonic line shape Eq. (3.10). For $\gamma=0.5 \omega_{0}$, the first anharmonic correction suffices to reproduce properly the numerical line-
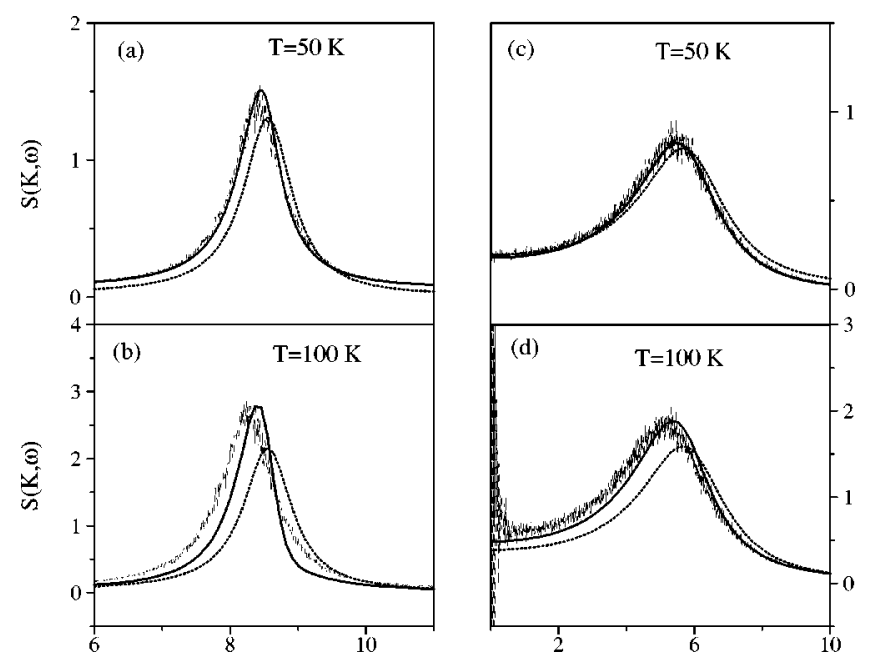

FIG. 4. Dynamic structure factor (line shape) of the $T$-mode peak for a Na adsorbate on the cosine potential, at two different temperatures. (a) and (b) $\gamma=0.1 \omega_{0}, V^{\ddagger}=134 \mathrm{meV}$. (c) and (d) $\gamma=0.5 \omega_{0}, V^{\ddagger}=67 \mathrm{meV}$. Dashed line: numerical simulation [see Eq. (2.1)]. Solid lines: anharmonic approximation, Fourier transform of Eqs. (3.18) and (3.19). Dotted lines: harmonic approximation, Fourier transform of Eq. (3.8).

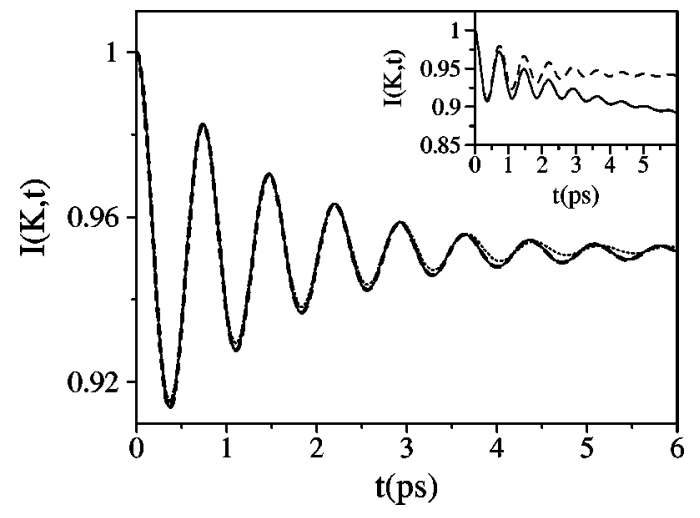

FIG. 5. Intermediate scattering function at $T=100 \mathrm{~K}, \gamma=0.1 \omega_{0}$ and $V^{\ddagger}$ $=67 \mathrm{meV}$. We have set $K=1 \AA^{-1}$. Dashed line: numerical simulation, Eq. (2.2). Solid line: Gaussian approximation, Eqs. (2.3) or (3.22), with the numerically calculated autocorrelation functions. Dotted line: numerical simulation of a quartic oscillator (fourth-order expansion of the cosine potential). Diffusing trajectories are removed from the autocorrelation functions. Inset: numerical (dashed) and Gaussian (solid) simulations taking into account diffusing trajectories.

shape until $T=100 \mathrm{~K}$, while in the low friction case good agreement is found only for the lower temperature of $T$ $=50 \mathrm{~K}$.

When deriving the intermediate scattering function or the line shape based on the leading-order contribution to the velocity correlation function, as in Eq. (3.17), one is making the additional assumption that the position or velocity are Gaussian processes (truncation of the cumulant expansion). In order to check this approximation, we calculate both the numerically exact intermediate scattering function through Eq. (2.2) and the Gaussian approximation Eqs. (2.3) or (3.22), using the numerically exact correlation functions. As seen in Fig. 5, the Gaussian approximation is an excellent one even in the case of low friction and low barrier values. Also, with the dotted line, we show the intermediate scattering function calculated for the quartic potential

$$
V(q)=\frac{1}{2} \omega_{0}^{2} q^{2}+K_{4} q^{4} .
$$

The good agreement suggests that indeed the first anharmonic term in the Taylor expansion of the adiabatic potential is able to account for the shift and broadening of the $T$-mode peak, and that the failure of the analytic theory at low barriers (high temperature) or low friction is mainly due to the fact that we neglected higher-order terms in the perturbative solution.

The curves in Figs. 3 and 5 have been obtained only with vibrational trajectories. For the parameters used in Fig. 5, especially at long times many of the trajectories overcome the barrier and start to diffuse (therefore giving also a quasielastic peak). In the Inset of Fig. 5 we show how the Gaussian approximation fails for the diffusing trajectories. Moreover, this approximation depends now on the value of the wave-vector transfer $K$ (it is better at small $K$ values), while the intermediate scattering function for the vibrational motion does not depend on $K$. Only its amplitude, and thus the intensity of the $T$-mode peak, will change, not its shape or position. 
From Fig. 4 we also note that the anharmonic dynamic structure factor presents a shift with temperature towards smaller frequencies. A calculation of the width from the analytical line shape, however, shows no significant broadening with temperature even in the range where it compares well to numerical simulations. This will be further discussed in Sec. IV.

\section{Quantum corrections}

As an example, we will work out the quantum line shape for the dissipative harmonic oscillator. Since quantum corrections are only significant at low temperatures, especially for adsorbates with a large mass, the harmonic approximation will suffice to illustrate the important quantum effects. It is convenient to express the Gaussian intermediate scattering function exactly ${ }^{30}$ in terms of the position autocorrelation function ${ }^{16}$ as

$$
I(K, t)=e^{-2 W} e^{K^{2}\langle q(t) q(0)\rangle} .
$$

The resultant dynamic structure factor will be real, showing two peaks at positive and negative frequencies (accounting for quantum creation and annihilation). Strictly, $S(K, \omega)$ and $S(K,-\omega)$ are related by a detailed balance condition preventing annihilation at $T=0,{ }^{30}$ therefore at low temperature the dynamic structure factor is asymmetric. The two peaks, however, will have nearly the same line shape (although their magnitudes can be very different). We can calculate an average line shape by replacing $\langle q(t) q(0)\rangle$ with its real part $C_{q}(t)=1 / 2[\langle q(0) q(t)\rangle+\langle q(t) q(0)\rangle]$.

To obtain the quantum position autocorrelation function we note that the quantum thermal average of the kinetic and potential energies are

$$
\frac{1}{2}\left\langle\lambda_{j}^{2} y_{j}^{2}(0)\right\rangle=\frac{1}{2}\left\langle p_{j}^{2}(0)\right\rangle=\frac{\hbar}{4} \lambda_{j} \operatorname{coth}\left(\hbar \beta \lambda_{j} / 2\right) .
$$

Using the normal-mode representation of the Hamiltonian one finds

$$
\begin{aligned}
C_{q}(t) & =\sum_{j=0}^{N} u_{j 0}^{2}\left\langle y_{j}^{2}(0)\right\rangle \cos \left(\lambda_{j} t\right) \\
& =\frac{\hbar}{\pi} \int_{0}^{\infty} d \lambda \Upsilon(\lambda) \operatorname{coth}(\hbar \beta \lambda / 2) \cos (\lambda t),
\end{aligned}
$$

where we have used the definition of the spectral density of the normal modes as given in Eq. (2.15). For Ohmic friction the spectral density is found from Eq. (2.17) so that one has

$$
C_{q}(t)=\frac{\hbar \gamma}{\pi} \int_{0}^{\infty} d \lambda\left(\frac{\lambda \operatorname{coth}(\hbar \beta \lambda / 2) \cos (\lambda t)}{\left(\omega_{0}^{2}-\lambda^{2}\right)^{2}+\gamma^{2} \lambda^{2}}\right) .
$$

Note that using $C_{q}(t)$ is consistent with the fact that the quantum fluctuation-dissipation theorem implicitly obeyed by the friction function here has the symmetrized form..$^{18,19}$

$$
\begin{aligned}
\gamma(t)= & \sum_{j=1}^{N} \frac{1}{2}\left\langle F_{r_{j}}(t) F_{r_{j}}(0)\right. \\
& \left.+F_{r_{j}}(0) F_{r_{j}}(t)\right\rangle \frac{2 \tanh \left(\hbar \omega_{j} \beta / 2\right)}{\hbar \omega_{j}} .
\end{aligned}
$$

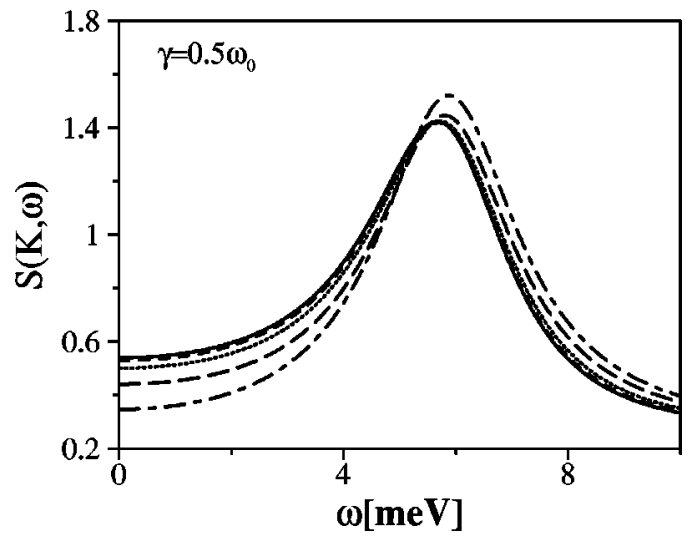

FIG. 6. Quantum dynamic structure factor in the harmonic approximation, from Eqs. (3.22) and (3.25), for the same parameters as in Figs. 4(c) and (d), $\gamma=0.5 \omega_{0}, V^{\ddagger}=67 \mathrm{meV}$, and several temperatures: solid line, classical result at $T=100 \mathrm{~K}$. Quantum results: dashed line, $T=100 \mathrm{~K}$; dotted line, $T$ $=50 \mathrm{~K}$; long dashed line, $T=25 \mathrm{~K}$; dot-dashed line, $T=10 \mathrm{~K}$. The wavevector transfer is $K=1 \AA^{-1}$.

The Debye-Waller factor for the harmonic oscillator is readily obtained by noting that

$$
\left\langle q^{2}(t)\right\rangle=\left\langle q^{2}(0)\right\rangle=C_{q}(0) .
$$

In Fig. 6 we compare the harmonic approximation for the classical and quantum dynamic structure factor for a $\mathrm{Na}$ atom moving on a cosine potential. The parameters chosen are $V^{*}=67 \mathrm{meV}$ and $\gamma=0.5 \omega_{0}$. Results are shown at several temperatures. One notes that lowering the temperature induces a blueshift of the $T$-mode peak. The observed experimental redshift is due to the anharmonicity as shall be shown below. Quantum effects decrease as the friction coefficient decreases, for $\gamma=0.1 \omega_{0}$, any effect is unobservable for temperatures above $10 \mathrm{~K}$.

\section{Multidimensional systems}

The same perturbation theory may be used for systems with more than one vibrational degree of freedom coupled to a bath. We will illustrate this for an atomic adsorbate on a two-dimensional periodic surface. The generalized Langevin equations for the two degrees of freedom problem take the form

$$
\begin{aligned}
& \ddot{q}+\frac{\partial V(q, z)}{\partial q}+\int_{0}^{t} d \tau \gamma_{q}(t-\tau) \dot{q}(\tau)=F_{q}(t), \\
& \ddot{z}+\frac{\partial V(q, z)}{\partial z}+\int_{0}^{t} d \tau \gamma_{z}(t-\tau) \dot{z}(\tau)=F_{z}(t),
\end{aligned}
$$

where the adiabatic atom-surface interaction potential $V(q, z)$ is generally nonseparable. ${ }^{3}$ The random forces $F_{q}$, $F_{z}$ are uncorrelated and obey the fluctuation-dissipation relations

$$
\left\langle F_{i}(t) F_{i}(0)\right\rangle=k_{B} T \gamma_{i}(t), \quad i=q, z .
$$

The Hamiltonian equivalent of the two coupled Langevin equations is ${ }^{25}$ 


$$
\begin{aligned}
H= & \frac{\left(p_{q}^{2}+p_{z}^{2}\right)}{2}+V(q, z)+\frac{1}{2} \sum_{j=1}^{N}\left[p_{q_{j}}^{2}+\left(\omega_{q_{j}} x_{j}-\frac{c_{q_{j}}}{\omega_{q_{j}}} q\right)^{2}\right] \\
& +\frac{1}{2} \sum_{j=1}^{N}\left[p_{z_{j}}^{2}+\left(\omega_{z_{j}} y_{j}-\frac{c_{z_{j}}}{\omega_{z_{j}}} z\right)^{2}\right] .
\end{aligned}
$$

The Hamiltonian representation for the two time-dependent frictions is

$$
\gamma_{i}(t)=\sum_{j=1}^{N} \frac{c_{i_{j}}^{2}}{\omega_{i_{j}}^{2}} \cos \left(\omega_{i_{j}} t\right), \quad i=q, z .
$$

The interaction potential $V(q, z)$ can be expanded around the minimum [for simplicity, located at the origin and with $V(0,0)=0]$ as

$$
V(q, z)=\frac{1}{2}\left[\omega_{q q}^{2} q^{2}+2 \omega_{q z} q z+\omega_{z z}^{2} z^{2}\right]+V_{1}(q, z),
$$

where $V_{1}(q, z)$ defines the anharmonic contributions. If we neglect them, the Hamiltonian may be again diagonalized using a normal-mode transformation; the orthogonal transformation matrix $\mathbf{U}$ has now dimensions $(2 N+2) \times(2 N$ $+2)$. The system coordinates $(q, z)$ are expressed in terms of the normal modes, see Eq. (2.13), as

$$
\begin{aligned}
& q=u_{-1-1} \rho+u_{0-1} \sigma+\sum_{j=1}^{2 N} u_{j-1} \nu_{j}, \\
& z=u_{-10} \rho+u_{00} \sigma+\sum_{j=1}^{2 N} u_{j 0} \nu_{j},
\end{aligned}
$$

where $\rho$ and $\sigma$ are the system normal modes corresponding to $q$ and $z$, respectively, and we have now $2 N$ bath modes $\nu_{j}$. The normal-mode Hamiltonian takes the form

$H_{N M}=\frac{1}{2}\left(p_{\rho}^{2}+p_{\sigma}^{2}+\lambda_{-1}^{2} \rho^{2}+\lambda_{0}^{2} \sigma^{2}\right)+\frac{1}{2} \sum_{j=1}^{2 N}\left(p_{\nu_{j}}^{2}+\lambda_{j}^{2} \nu_{j}^{2}\right)$.

Equation (2.16) can be generalized to the multidimensional case: ${ }^{26}$

$$
\begin{aligned}
& \sum_{j=-1}^{2 N} \frac{u_{j-1}^{2}}{s^{2}+\lambda_{j}^{2}} \\
& =\frac{s^{2}+s \hat{\gamma}_{z}(s)+\omega_{z z}^{2}}{\left[s^{2}+s \hat{\gamma}_{q}(s)+\omega_{q q}^{2}\right]\left[s^{2}+s \hat{\gamma}_{z}(s)+\omega_{z z}^{2}\right]-\omega_{q z}^{2}}, \\
& \sum_{j=-1}^{2 N} \frac{u_{j-1} u_{j 0}}{s^{2}+\lambda_{j}^{2}} \\
& =\frac{-\omega_{q z}}{\left[s^{2}+s \hat{\gamma}_{q}(s)+\omega_{q q}^{2}\right]\left[s^{2}+s \hat{\gamma}_{z}(s)+\omega_{z z}^{2}\right]-\omega_{q z}^{2}}, \\
& \sum_{j=-1}^{2 N} \frac{u_{j 0}^{2}}{s^{2}+\lambda_{j}^{2}} \\
& =\frac{s^{2}+s \hat{\gamma}_{q}(s)+\omega_{q q}^{2}}{\left[s^{2}+s \hat{\gamma}_{q}(s)+\omega_{q q}^{2}\right]\left[s^{2}+s \hat{\gamma}_{z}(s)+\omega_{z z}^{2}\right]-\omega_{q z}^{2}} .
\end{aligned}
$$

To simplify matters, we will obtain the velocity autocorrelation function for a symmetric surface with Ohmic friction, such as the $\mathrm{Cu}(001)$ system. ${ }^{3}$ Then $\gamma_{q}=\gamma_{z} \equiv \gamma, \omega_{q q}=\omega_{z z}$ $\equiv \omega_{0}, \omega_{q z}=0$, and $\langle\dot{q}(t) \dot{q}(0)\rangle=\langle\dot{z}(t) \dot{z}(0)\rangle$. From the equations of motion for the normal-mode coordinates given by $H_{\mathrm{NM}}$, and Eqs. (3.35)-(3.37), one sees that for a symmetric surface the harmonic line shape is identical to the onedimensional case. The first anharmonic correction to the potential is

$$
V_{1}(q, z)=K_{4}^{q} q^{4}+K_{4}^{z} z^{4}+2 K_{4}^{q z} q^{2} z^{2},
$$

since the surface is symmetric one has that $K_{4}^{q}=K_{4}^{z} \equiv K_{4}$. A perturbative solution to first order in $K_{4}$ and $K_{4}^{q z}$ for the normal-mode coordinates is found to be

$$
\nu_{j}(t)=\nu_{j}^{(0)}(t)+4 K_{4} \nu_{j}^{(1)}(t)+4 K_{4}^{q z} \mu_{j}^{(1)}(t),
$$

where

$$
\begin{aligned}
\nu_{j}^{(1)}(t)= & -\frac{u_{j-1}}{\lambda_{j}} \int_{0}^{t} \sin \left[\lambda_{j}\left(t-t^{\prime}\right)\right] F_{-1}\left(t^{\prime}\right) d t^{\prime} \\
& -\frac{u_{j 0}}{\lambda_{j}} \int_{0}^{t} \sin \left[\lambda_{j}\left(t-t^{\prime}\right)\right] F_{0}\left(t^{\prime}\right) d t^{\prime}
\end{aligned}
$$

with

$$
F_{i}(t) \equiv\left(\sum_{j=-1}^{2 N} u_{j i} \nu_{j}^{(0)}(t)\right)^{3}, \quad i=-1,0,
$$

and

$$
\begin{aligned}
\mu_{j}^{(1)}(t)= & -\frac{u_{j-1}}{\lambda_{j}} \int_{0}^{t} \sin \left[\lambda_{j}\left(t-t^{\prime}\right)\right] G_{-1}\left(t^{\prime}\right) d t^{\prime} \\
& -\frac{u_{j 0}}{\lambda_{j}} \int_{0}^{t} \sin \left[\lambda_{j}(t-t)^{\prime}\right] G_{0}\left(t^{\prime}\right) d t^{\prime}
\end{aligned}
$$

with

$$
G_{-1}(t) \equiv\left(\sum_{j=-1}^{2 N} u_{j-1} \nu_{j}^{(0)}(t)\right)\left(\sum_{j=-1}^{2 N} u_{j 0} \nu_{j}^{(0)}(t)\right)^{2}
$$

and the subindices 0 and -1 are interchanged for $G_{0}(t)$.

Although more cumbersome, it is straightforward, using the thermal averages (3.14) and the expressions (3.35)(3.37), to calculate the anharmonic autocorrelation function. Its Laplace transform reads [compare to Eq. (3.16)]

$$
\begin{aligned}
\left\langle\hat{p}_{q}(s) \hat{p}_{q}(0)\right\rangle= & \frac{1}{\beta} \frac{s}{s^{2}+s \hat{\gamma}(s)+\omega_{0}^{2}} \\
& -\left(\frac{12 K_{4}+4 K_{4}^{q z}}{\beta^{2} \omega_{0}^{2}}\right) \frac{s}{\left[s^{2}+s \hat{\gamma}(s)+\omega_{0}^{2}\right]^{2}} .
\end{aligned}
$$

For a symmetric surface, such as for the $\mathrm{Cu}(001)$ system, one can model the two-dimensional (2D) PES as ${ }^{31}$ 


$$
\begin{aligned}
V(x, y)= & V_{0}-V_{1}\left[\cos \left(\frac{2 \pi x}{a}\right)+\cos \left(\frac{2 \pi y}{a}\right)\right] \\
& +V_{2} \cos \left(\frac{2 \pi x}{a}\right) \cos \left(\frac{2 \pi y}{a}\right) .
\end{aligned}
$$

Choosing $V_{0}, V_{1}$, and $V_{2}$ such that $V(0,0)=0, V(0, \pm a / 2)$ $=V^{\ddagger}$ (barrier energy at the saddle point) and $V( \pm a / 2$, $\pm a / 2)=V_{m}$ (energy barrier at the maxima) results in $V_{0}$ $=V_{m} / 4+V^{\ddagger} / 2, V_{1}=V_{m} / 4$ and $V_{2}=V_{m} / 4-V^{\ddagger} / 2$. With this choice for the adiabatic PES, $K_{4}=-\omega_{0}^{4} / 12 V^{\ddagger}$ is the same as for the one-dimensional case, and

$$
K_{4}^{q z}=\frac{\omega_{0}^{4}}{12 V^{\ddagger}}\left(\frac{V_{m}}{2 V^{\ddagger}}-1\right) .
$$

Note that for $V_{m}=2 V^{*}$ the potential is separable and identical to the 1D case. In general $\left|K_{4}^{q z}\right|<\left|K_{4}\right|$ (for instance, in Ref. 31 a fitting to the QHAS experiments gave the value $\left.V_{m} / V^{\ddagger}=5 / 4\right)$ and thus the coupling between the surface coordinates has only a small effect on the $T$-mode peak. We have seen from the analytical estimate using Eq. (3.44) and from numerical simulations that the line shape does not change significantly for this particular model and coupling. This does not mean that, experimentally, one cannot distinguish between a separable and nonseparable adiabatic surface: the quasielastic peak is sensitive to the multidimensional features of the surface. The nonseparability can have a large effect on the curvature close to the barrier, which enters in the multidimensional Kramers prefactor, and this does affect the diffusion significantly. ${ }^{9}$

\section{TEMPERATURE-DEPENDENT SHIFT AND BROADENING}

Frequency shifts can be obtained directly with the preceding formalism without calculating the dynamic structure factor. From Eq. (3.11) one notes that the anharmonicity will cause a shift of both the minimum and the frequency of the system normal mode. Specifically, the instantaneous frequency, to leading order in the anharmonicity parameter $K_{4}$ is

$$
\begin{aligned}
\lambda_{0}(t) & \equiv\left(\frac{\partial^{2} H}{\partial \rho^{2}}\right)^{1 / 2} \\
& =\left[\lambda_{0}^{2}+12 K_{4} u_{00}^{2}\left(\sum_{j=0}^{N} u_{j 0} y_{j}\right)^{2}\right]^{1 / 2} \\
& \sim \lambda_{0}+\frac{6 K_{4} u_{00}^{2}}{\lambda_{0}}\left(\sum_{j=0}^{N} u_{j 0} y_{j}\right)^{2}+o\left(K_{4}^{2}\right) .
\end{aligned}
$$

The frequency shift is obtained from the thermal average $\left\langle\lambda_{0}(t)\right\rangle$. Introducing the harmonic oscillator solution, Eq. (3.4), for the normal-mode coordinate $y_{j}$ in the last term in Eq. (4.1), and performing the averages (ignoring the anharmonicity in the partition function) we find a temperaturedependent contribution to the frequency shift

$$
\Delta \bar{\lambda}_{0} \equiv\left\langle\lambda_{0}(t)\right\rangle-\lambda_{0}=\frac{6 K_{4}}{\beta \omega_{0}^{2} \omega_{1}} .
$$

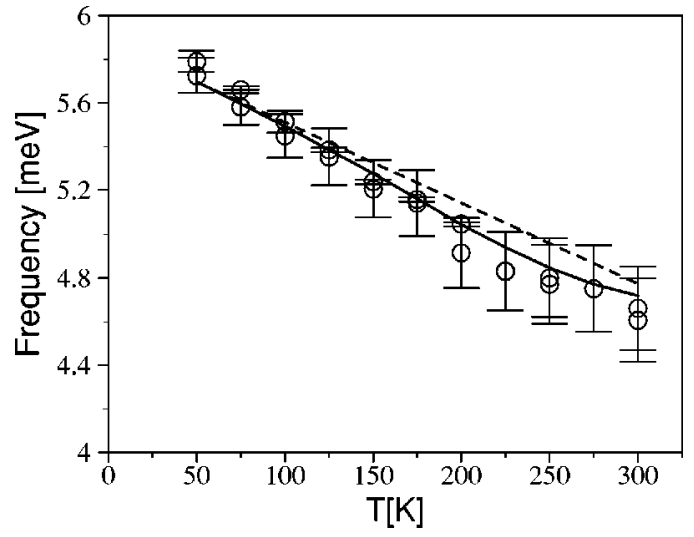

FIG. 7. T-mode peak position as a function of temperature for the $\mathrm{Na} /$ $\mathrm{Cu}(001)$ system. Circles: experimental results, Ref. 3. Solid line: numerical results obtained by solving the Langevin equation with the $1 \mathrm{D}$ cosine potential (3.1). The parameters used, $\gamma=0.1 \omega_{0}$ and $V^{\ddagger}=75 \mathrm{meV}$, have been estimated from experiment (Ref. 3). Dashed line: theoretical prediction, Eq. (4.2), using first-order anharmonic corrections.

This is the result given in Eq. (1.2) keeping only the firstorder correction. As already noted above, for the cosine potential $K_{4}$ is negative, so that the anharmonicity produces a redshift with a linear temperature dependence.

In Fig. 7 we compared the shift obtained from the normal-mode frequency average, Eq. (4.2), with numerical (solid lines) and experimental results obtained for the $\mathrm{Na}$ / $\mathrm{Cu}(001)$ system (taken from Ref. 3). The value of the barrier and friction coefficient estimated from experimental measurements are $V^{\ddagger} \sim 75 \mathrm{meV}$ and $\gamma / \omega_{0}=0.1$. The agreement is fairly good for temperatures below $T \sim 150 \mathrm{~K}$. We will show below that, due to the low friction of the system and the relatively small barrier, second-order corrections in the anharmonicity are necessary for higher temperatures.

In the analytical estimation of the lineshape given in Sec. III B, a shift was indeed observed but there was no broadening. This can be understood by calculating the variance in the system frequency, $\left\langle\left[\lambda_{0}(t)-\left\langle\lambda_{0}(t)\right\rangle\right]^{2}\right\rangle$, which is expected to be proportional to the broadening of the peak. One immediately sees that, to first order in $K_{4}$, the variance vanishes. Therefore second-order corrections in the anharmonicity are necessary for obtaining the temperature-dependent broadening. A direct calculation of the dynamic structure factor along the lines discussed in Sec. III B, using second-order perturbation theory, is rather involved. Instead we resort to a simpler model which, however, retains the basic dynamical ingredients: the Kubo oscillator. ${ }^{14,32}$ First, we expand the intermediate scattering function (2.2) in moments, instead of cumulants. If we remain to order 2 , this is equivalent to a second-order Taylor expansion of Eq. (3.22) around $K=0$. Since the $T$-mode peak shape is independent of $K$ the moment expansion is at least in principle, convergent. Then the intermediate scattering function will be essentially proportional to the position autocorrelation function, as in the theory for Raman scattering, ${ }^{33}$ and we can use the Kubo oscillator model.

We assume that the normal-mode oscillator with frequency $\lambda_{0}$ has a random frequency component due to the anharmonicity, and define 


$$
\lambda_{0}(t)=\lambda_{0}+\lambda_{1}(t) .
$$

Then, using the equations of motion of an oscillator with frequency $\lambda_{0}(t)$ and a cumulant expansion to second order we find

$$
\begin{aligned}
\langle q(t) q(0)\rangle & \propto \operatorname{Re}\left\langle e^{i \int_{0}^{t} \lambda_{1}\left(t^{\prime}\right) d t^{\prime}}\right\rangle e^{i \lambda_{0} t} \\
& \sim \operatorname{Re} e^{i \lambda_{0} t} e^{i \int_{0}^{t}\left\langle\lambda_{1}\left(t^{\prime}\right)\right\rangle d t^{\prime}} e^{-\int_{0}^{t}\left(t-t^{\prime}\right)\left\langle\lambda_{1}\left(t^{\prime}\right) \lambda_{1}(0)\right\rangle d t^{\prime}}
\end{aligned}
$$

Note that $\left\langle\lambda_{1}\left(t^{\prime}\right)\right\rangle$ is the shift $\Delta \bar{\lambda}_{0}$, Eq. (4.2). Using Eq. (2.20) for $\lambda_{0}$ we can express the intermediate scattering function as

$I(K, t) \propto \operatorname{Re} e^{i\left(\omega_{1}+\Delta \bar{\lambda}_{0}\right) t} e^{-\gamma t / 2} e^{-\int_{0}^{t}\left(t-t^{\prime}\right)\left\langle\lambda_{1}\left(t^{\prime}\right) \lambda_{1}(0)\right\rangle d t^{\prime}}$.

To first order in $K_{4}$ one sees that $\left\langle\lambda_{1}\left(t^{\prime}\right) \lambda_{1}(0)\right\rangle=0$ and the dynamic structure factor is a Lorentzian centered at the frequency $\omega_{1}+\Delta \bar{\lambda}_{0}$ (and thus there is a linear temperaturedependent shift), with a FWHM equal to the harmonic oscillator width $\gamma$ (and thus no temperature-dependent broadening). Second-order corrections in $\lambda_{0}(t)$ will produce a temperature-dependent broadening due to the last exponential factor in Eq. (4.5) above. To second order, the instantaneous frequency is given by

$$
\begin{aligned}
\lambda_{0}(t) \sim & \lambda_{0}+\frac{6 K_{4} u_{00}^{2}}{\lambda_{0}}\left[\left(\sum_{j=0}^{N} u_{j 0} y_{j}^{(0)}\right)^{2}\right. \\
& \left.+8 K_{4}\left(\sum_{j=0}^{N} u_{j 0} y_{j}^{(0)}\right)\left(\sum_{j=0}^{N} u_{j 0} y_{j}^{(1)}\right)\right] \\
& -\frac{18 K_{4}^{2} u_{00}^{4}}{\lambda_{0}^{3}}\left(\sum_{j=0}^{N} u_{j 0} y_{j}^{(0)}\right)^{4}+o\left(K_{4}^{3}\right),
\end{aligned}
$$

where we have now introduced the first-order perturbative solution $y_{j}^{(1)}$, Eq. (3.12). For the correlation function $\left\langle\lambda_{1}\left(t^{\prime}\right) \lambda_{1}(0)\right\rangle$ terms depending on $y_{j}^{(1)}$ cancel out and we obtain terms depending only on averages over the harmonic oscillator position process. Since this is a Gaussian process (for analytic simplicity we ignore the anharmonicity in the partition function), we use the well-known identities

$$
\begin{aligned}
& \left\langle q_{\mathrm{osc}}^{2}(t)\right\rangle=\left\langle q_{\mathrm{osc}}^{2}(0)\right\rangle=\frac{1}{\beta \omega_{0}^{2}}, \\
& \left\langle q_{\mathrm{osc}}^{2}(t) q_{\mathrm{osc}}^{2}(0)\right\rangle=2\left\langle q_{\mathrm{osc}}(t) q_{\mathrm{osc}}(0)\right\rangle^{2}+\left\langle q_{\mathrm{osc}}^{2}(0)\right\rangle^{2}
\end{aligned}
$$

so that everything can be cast in terms of the average $\left\langle q_{\text {osc }}^{2}(0)\right\rangle$ and the position correlation function $\left\langle q_{\text {osc }}(t) q_{\text {osc }}(0)\right\rangle$ which are known. We then find that

$$
\begin{aligned}
\left\langle\lambda_{1}\left(t^{\prime}\right) \lambda_{1}(0)\right\rangle= & 36 K_{4}^{2} \frac{u_{00}^{4}}{\lambda_{0}^{2}}\left\langle q_{\text {osc }}^{2}(0)\right\rangle^{2} \\
& +72 K_{4}^{2} \frac{u_{00}^{4}}{\lambda_{0}^{2}}\left\langle q_{\text {osc }}(t) q_{\text {osc }}(0)\right\rangle^{2} .
\end{aligned}
$$

Substituting Eq. (4.9) and [see Eq. (3.24)]

$$
\left\langle q_{\mathrm{osc}}(t) q_{\mathrm{osc}}\right\rangle=\frac{e^{-\gamma t / 2}}{\beta \omega_{0}^{2}}\left[\cos \left(\omega_{1} t\right)+\frac{\gamma}{2 \omega_{1}} \sin \left(\omega_{1} t\right)\right]
$$

one can readily calculate the integrals in the last exponent in Eq. (4.5).

We note that, for long times, the first term in Eq. (4.9) is dominant and gives a Gaussian intermediate scattering function of the form

$$
I(K, t) \propto \operatorname{Re} e^{i\left(\omega_{1}+\Delta \bar{\lambda}_{0}\right) t} e^{-\gamma t / 2} e^{-\sigma^{2}\left(t^{2} / 2\right)},
$$

where

$$
\sigma=\frac{6\left|K_{4}\right|}{\beta \omega_{1} \omega_{0}^{2}}=\frac{\omega_{0}^{2}}{2 \beta V^{\ddagger} \omega_{1}} .
$$

The dynamic structure factor is the time Fourier transform of Eq. (4.11). The imaginary exponential gives the peak position, already discussed above. The width of the peak is determined by the real exponentials. At short times, or if $\beta V^{\ddagger}$ $\gg 1$ [see Eq. (4.12)], the first exponent dominates, the peak has a Lorentzian shape with the FWHM given mainly by $\gamma$, as in the harmonic oscillator case. At long times, or if $\beta V^{\ddagger}$ is small, the Gaussian contribution dominates and the peak has a Gaussian shape with a FWHM mainly given by $\sigma$. In intermediate situations both contributions have to be taken into account, the central part of the $T$-mode peak being closer to a Gaussian while the wings are better approximated with a Lorentzian shape. This is analogous to the motional narrowing effect, ${ }^{14,34}$ first discussed in the context of magnetic resonance absorption. It is important to note that this simple model gives a width proportional to $\sigma$, and therefore a linear temperature-dependent broadening.

In Fig. 2 we compared the experimental broadening with the numerical one, and the full width at half maximum obtained from the Fourier transform of the Kubo intermediate scattering function, Eq. (4.5). This simple model is able to account for the linear increase of the broadening with temperature as observed experimentally.

Second-order corrections can also be calculated for the shift using Eq. (4.6), with the result

$$
\Delta \bar{\lambda}_{0}=\frac{6 K_{4}}{\beta \omega_{0}^{2} \omega_{1}}-\frac{54 K_{4}^{2}}{\beta^{2} \omega_{0}^{6} \omega_{1}},
$$

which gives Eq. (1.2). For the $\mathrm{Na} / \mathrm{Cu}(001)$ system, these corrections are important for $T>100 \mathrm{~K}$ and now the agreement with numerical and experimental results is better for the high-temperature range, see Fig. 1. In this figure we also plot with dotted lines the quantum-mechanical shift obtained along the lines sketched in Sec. III C. The shift depends finally on averages of the type $\left\langle q_{\text {osc }}^{2}(0)\right\rangle \equiv C_{q}(0)$ so these may be readily calculated from Eq. (3.25) with $t=0$. For this particular system quantum effects are experimentally unobservable for the temperature range spanned. We do note that at low temperatures the quantum-mechanical blueshift compensates for the redshift induced by the anharmonicity.

\section{CONCLUSIONS}

In this work we presented an analytical theory for the line shape, shift, and broadening of the $T$-mode peak based on two assumptions: the first one is that the dynamics of isolated adsorbates is well reproduced by a generalized Langevin equation, and the second assumption is that the 
first anharmonic correction of the interaction potential suffices to account for the temperature dependence. The validity of the first assumption has been demonstrated for several atom-surface systems at low coverages, where numerical simulations have been contrasted to results of QHAS experiments. ${ }^{3,9,29}$ In this paper we have demonstrated that also the second assumption is correct for systems even in the low friction regime if $\beta V^{\ddagger} \gg 1$. The theory is based on a normal-mode transformation of the Hamiltonian equivalent of the Langevin equation.

For the line shape we used a first-order perturbative solution of the normal-mode coordinates. The line shape is Lorentzian only in the harmonic approximation, first-order anharmonic corrections produce a temperature-dependent shift and asymmetry of the peak. The agreement with numerical simulations is good for $\beta V^{\ddagger} \sim 30$ in the low friction range $\left(\gamma=0.1 \omega_{0}\right)$, and $\beta V^{\ddagger} \sim 8$ in the intermediate friction regime $\left(\gamma=0.5 \omega_{0}\right)$. For the shift and broadening, we have employed a perturbative expansion in the instantaneous system frequency. First-order corrections suffice to account for a linear temperature-dependent shift. To obtain the temperature dependence of the broadening, we had to include secondorder corrections. Simple analytic expressions for the shift and width were derived using the Kubo oscillator model. These reproduce quantitatively (without any fitting of the parameters) the numerical and experimental shift and broadening of the $T$-mode peak, and show also a motional narrowing effect between Lorentzian and Gaussian shapes as the reduced barrier height $\left(\beta V^{\ddagger}\right)$ is decreased.

Quantum corrections may play a role for damped adsorbates at low temperatures (or for adsorbates with small mass). For a symmetric surface the quantum shift is opposite in sign to the shift induced by the anharmonicity. We have also extended the theory to the case of an adsorbate on a two-dimensional surface with coupled degrees of freedom, but the effect of the coupling is typically small in the $T$-mode shape and position.

One of the useful consequences, for experiments, of the theory presented here is that one could estimate the anharmonicity parameter from the $T$-mode shift, using Eqs. (4.2) and (4.13). Among the systems studied so far by QHAS techniques, the $\mathrm{Na} / \mathrm{Cu}(001)$ is an ideal candidate to test our predictions, since many experimental results and accurate estimations of barrier heights and frequencies are available at low coverages. From the comparison with experiment we indeed show that the theory correctly predicts the temperature dependence of the $T$-mode position. From the anharmonicity parameter one can also estimate the barrier height, providing additional information which can be contrasted to that obtained by the analysis of the quasielastic peak. Other adsorbate-surface systems whose $T$-mode features have been studied by QHAS techniques are the $\mathrm{Xe} / \mathrm{Pt}(111)$ and $\mathrm{Na} /$ $\mathrm{Pt}(111)$ systems, and especially the $\mathrm{CO}$ molecule adsorbed on different substrates, such as $\mathrm{Ni}(110), \mathrm{Cu}(001)$, and Pt(111) (for a compilation of QHAS data see Ref. 8 and references therein). Most of these systems have small friction values and barriers below $70 \mathrm{meV}$.

Of special interest to our discussion is the $\mathrm{CO} / \mathrm{Pt}(111)$ system, which has an estimated friction value $\gamma \sim 0.1 \omega_{0}$ and a diffusion barrier whose height is $V^{\ddagger} \sim 130 \mathrm{meV}$. ${ }^{4}$ Experimental measurements on this system show a small $T$-mode frequency shift with temperature, but to within experimental accuracy, the width of the peak is temperature independent. This is not very surprising in view of the high barrier for this particular system and our previous conclusions from the anharmonic theory. However, in contrast to the $\mathrm{Na} / \mathrm{Cu}(001)$ system, here one finds that the $T$-mode frequency shifts to the blue with increasing temperature. There is, however, evidence of a strong coupling between the $T$ mode and the $\mathrm{CO}$ rotation, as in the $\mathrm{CO} / \mathrm{Cu}(001)$ case $^{5}$ which changes the symmetry of the PES and could be responsible for the opposite shift. It is worth mentioning that another source of difficulty is present when analyzing experiments, especially at high temperatures, since vibrational and diffusional motions can not be always well separated.

To conclude, we remark that the Hamiltonian formalism has been successfully employed for the analysis of both the quasielastic $^{9}$ and the $T$-mode peaks in QHAS experiments. In the first case, one has to consider the dynamics at energies close to the diffusion barrier. The relevant physical quantity is the normal-mode system frequency at the barrier, which is imaginary and gives the Kramers prefactor ${ }^{20}$ therefore playing the role of an attempt frequency for escaping the potential well. For the analysis of the $T$-mode peak we are interested in the dynamics at the well, and the normal-mode system frequency is complex. The real part gives the peak position at zero temperature, including the shift in the oscillator frequency due to the coupling to the bath. The imaginary part gives the width at zero temperature, which is the friction coefficient measuring the rate of energy transfer of the oscillator to the bath.

Finally, we note that the results presented here can be improved in several aspects. One is the calculation of the second-order corrections in the line shape as obtained from Eq. (2.3), for a direct comparison to the experimental line shape at higher temperatures. The other aspect is the more accurate evaluation of the normal-mode averages, which we obtained by steepest descent. This is analogous to the inclusion of finite barrier corrections in the Kramers problem. ${ }^{25}$

\section{ACKNOWLEDGMENTS}

This work has been supported in part by DGICYT (Spain) under Contract No. BFM2001-2179. R.G. and J.L.V. thank the Ministry of Science and Technology (Spain) for a Ramón y Cajal Contract and a predoctoral F.P.I. grant, respectively. This work has also been supported by grants from the Israel Science Foundation, the Minerva Foundation (Munich), and the Volkswagen Foundation.

\footnotetext{
${ }^{1}$ F. Hofmann and J. P. Toennies, Chem. Rev. 96, 1307 (1996).

${ }^{2}$ A. P. Graham, F. Hofmann, and J. P. Toennies, J. Chem. Phys. 104, 5311 (1996).

${ }^{3}$ A. P. Graham, F. Hofmann, J. P. Toennies, L. Y. Chen, and S. C. Ying, Phys. Rev. Lett. 78, 3900 (1997); Phys. Rev. B 56, 10567 (1997).

${ }^{4}$ A. P. Graham and J. P. Toennies, Europhys. Lett. 42, 449 (1998).

${ }^{5}$ A. P. Graham, F. Hofmann, J. P. Toennies, G. P. Williams, C. J. Hirschmugl, and J. Ellis, J. Chem. Phys. 108, 7825 (1998).

${ }^{6}$ B. H. Choi, A. P. Graham, K. T. Tang, and J. P. Toennies, J. Chem. Phys. 116, 7695 (2002).
} 
${ }^{7}$ B. N. J. Persson and R. Ryberg, Phys. Rev. B 32, 3586 (1985).

${ }^{8}$ A. P. Graham and J. P. Toennies, Surf. Sci. 427-428, 1 (1999).

${ }^{9}$ R. Guantes, J. L. Vega, S. Miret-Artés, and E. Pollak, J. Chem. Phys. 119, 2780 (2003).

${ }^{10}$ B. N. J. Persson, E. Tosatti, D. Fuhrmann, G. Witte, and Ch. Wöll, Phys. Rev. B 59, 11777 (1999).

${ }^{11}$ B. N. J. Persson, Chem. Phys. Lett. 149, 278 (1988).

${ }^{12}$ L. van Hove, Phys. Rev. 95, 249 (1954).

${ }^{13}$ J. W. M. Frenken and B. J. Hinch, in Helium Atom Scattering from Surfaces, edited by E. Hulpke, Springer Series in Surface Sciences No. 27 (Springer-Verlag, New York, 1992), p. 287.

${ }^{14}$ R. Kubo, T. Toda, and N. Hashitsume, Statistical Physics II (SpringerVerlag, Berlin, 1991).

${ }^{15}$ J. L. Vega, R. Guantes, and S. Miret-Artés, J. Phys.: Condens. Matter 14, 6193 (2002).

${ }^{16}$ J. R. Manson and V. Celli, Phys. Rev. B 39, 3605 (1989).

${ }^{17}$ H. Risken, The Fokker-Planck Equation (Springer, Berlin, 1989).

${ }^{18}$ A. O. Caldeira and A. J. Leggett, Ann. Phys. (N.Y.) 149, 374 (1983).

${ }^{19}$ E. Cortés, B. J. West, and K. Lindenberg, J. Chem. Phys. 82, 2708 (1985).
${ }^{20}$ E. Pollak, J. Chem. Phys. 85, 865 (1986); Phys. Rev. A 33, 4244 (1986).

${ }^{21}$ A. M. Levine, M. Shapiro, and E. Pollak, J. Chem. Phys. 88, 1959 (1988).

${ }^{22}$ I. Rips and E. Pollak, Phys. Rev. A 41, 5366 (1990).

${ }^{23}$ E. Pollak in Dynamics of Molecules and Chemical Reactions, edited by R. E. Wyatt and J. Z. H. Zhang (Dekker, New York, 1996).

${ }^{24}$ E. Pollak, H. Grabert, and P. Hänggi, J. Chem. Phys. 91, 4073 (1989).

${ }^{25}$ A. M. Berezhkovskii, E. Pollak, and V. Y. Zitserman, J. Chem. Phys. 97, 2422 (1992)

${ }^{26}$ E. Hershkovitz and E. Pollak, J. Chem. Phys. 106, 7678 (1997).

${ }^{27}$ L. Y. Chen and S. C. Ying, Phys. Rev. Lett. 71, 4361 (1993).

${ }^{28}$ J. Ellis and J. P. Toennies, Surf. Sci. 317, 99 (1994).

${ }^{29}$ J. Ellis and J. P. Toennies, Phys. Rev. Lett. 70, 2118 (1993).

${ }^{30}$ See, for instance, S. W. Lovesey, Theory of Neutron Scattering from Condensed Matter (Clarendon, Oxford, 1984), Vol. 1, Chap. 3.

${ }^{31}$ J. Ellis, A. P. Graham, F. Hofmann, and J. P. Toennies, Phys. Rev. B 63, 195408 (2001).

${ }^{32}$ R. Kubo, J. Math. Phys. 4, 174 (1963).

${ }^{33}$ R. G. Gordon, Adv. Magn. Reson. 3, 1 (1968).

${ }^{34}$ J. H. Van Vleck, Phys. Rev. 74, 1168 (1948). 